\title{
Benefits of an older persons' assessment and liaison team in acute admissions areas of a general hospital
}

This article was published in the following Dove Press journal:

Pragmatic and Observational Research

21 August 2010

Number of times this article has been viewed

\section{Stephen Allen \\ Tom Bartlett \\ Joanna Ventham \\ Cherry McCubbin \\ Andrew Williams}

The Royal Bournemouth Hospital, Bournemouth, Dorset, United Kingdom
Correspondence: S C Allen

Consultant Physician and Professor of Clinical Gerontology, The Royal

Bournemouth Hospital, Castle Lane East, Bournemouth, Dorset, BH7 7DW, UK

Tel +440120270 4539

Fax +440I20270 4542

Email stephen.allen@rbch.nhs.uk
Objective: To analyze and describe the operational benefits that followed the introduction of a multiprofessional older person assessment and liaison service (OPAL) into the acute admissions areas of a general hospital. OPAL delivered comprehensive geriatric assessment and a range of early medical, nursing, therapy, and social interventions to all eligible elderly and frail patients.

Methods: A mix of numeric data, case note narrative, historic comparison, and staff opinion was used to reach a reliable view of the impact that OPAL had on a number of key indicators pertaining to the timing of assessments, treatments, and discharge planning.

Results: We found that the new service reduced the time required to achieve several critical interventions including medical, nursing, and therapy reviews. We were also able to show that OPAL activity played a critical role in reducing the length of hospital stay of frail older people and made available the equivalent of $9-16$ beds per day ( $8 \%-14 \%$ of acute admission area beds).

Conclusion: OPAL was shown to be effective as a medium for timely review and intervention of frail elderly patients in an acute medical setting, and as a mechanism for reducing length of stay.

Keywords: older persons, elderly, frail, early supported discharge, multiprofessional, flexible working

\section{Introduction}

In 2003 we conducted a large clinical study of acute medical admissions to a general hospital. The findings of that study and the potential it created for understanding the needs of acutely ill elderly people were subsequently published. ${ }^{1}$ This formed the basis of a bid for resources to provide early high-quality multidisciplinary input to expedite the diagnosis, treatment, and discharge planning needs of frail older patients presenting to the hospital as emergencies. After a series of negotiations between the Acute Trust and its Primary Care Trust, an agreement on funding for a new service was reached. This became fully operational in 2007 and was called the Older Persons Assessment and Liaison (OPAL) team, having been modeled to some extent on the innovative service of that name described by Harari et al. ${ }^{2}$ In 2009 we performed another large clinical study of acute medical admissions to compare with the data from 2003. There were several reasons for repeating the study, one of which was to generate data to provide numeric and narrative information on the impact of OPAL. Time scales, resources, managerial imperatives, and ethical considerations had precluded introducing OPAL as part of a controlled trial, so an alternative approach to gauge the effects of the new service was necessary. Another intention was to establish the current profile of medical admissions that formed the context within which OPAL operated. 
We anticipated considerable difficulties in measuring the impact of a newly introduced service that was embedded alongside other complex and variable systems of care. This was particularly the case because implementation had occurred stepwise, and some aspects of the operational policy had been modified in the light of feedback and experience. Service activity data provided information about the overall workload of OPAL and enabled a tally to be made of the number of patients for whom ward admission (beyond the acute admissions areas) had been avoided but gave little indication of the effectiveness of the team in terms of length of stay, capture of appropriate cases, or speed of access to services within the hospital. The detailed information collected in the 2003 and 2009 studies of acute medical admissions provided an opportunity for us to compare the care journeys of matched cases before OPAL and once it was fully functioning in a steady operational state. Fortunately, most other aspects of the acute medical admissions service that might influence the management of frail elderly patients had changed little during that time period, so it was reasonable to assume that most of any apparent effect of OPAL demonstrated was unlikely to be due mainly to other factors. Further, there were no substantial changes in the range or volume of community services available before and after the advent of OPAL.

We set out to answer the following preliminary questions:

- What was the mean daily acute medical admission rate in 2009?

- What was the proportion of admissions in 2009 that met the working definition for elderly and/or frail?

- Was the initial triage of elderly patients appropriate?

- What was the proportion of eligible patients reviewed by the OPAL team?

We then conducted:

- A collation of overall OPAL activity from managerial records for the corresponding half-year period

- Length of stay analyses of patients treated by OPAL in the Accident and Emergency Department, and after medical admission

- A detailed analysis of the timeliness of the components of OPAL

- An estimation of the bed-days released by OPAL activity

- An estimation of medical admissions avoided as a result of OPAL intervention in the Accident and Emergency Department

- Informal interviews with OPAL staff, other staff with operational proximity to OPAL, and managers to record their views regarding the reasons for the perceived success of the new service
- An appraisal of the personal and professional characteristics that enabled the OPAL team to function as originally conceived.

\section{OPAL team}

It was clear from the outset that the OPAL team would need to work flexibly, confidently, and cooperatively in order to deliver the expected combination of high-quality care and reduced length of stay in hospital. From our own pilot work, and the experiences of others, certain personal characteristics were seen as likely critical success factors for all OPAL staff, which were then embedded in person specifications and interview criteria. These included:

- Commitment to the OPAL project

- High levels of personal and professional maturity

- An holistic problem-solving approach to patient care

- Willingness to make decisions and take calculated risks

- Ability to "make things happen".

A phase of careful recruitment was undertaken to assemble the full team, which became fully operational in June 2007. The team consisted of:

- Two full-time registered nurses

- Three full-time occupational therapists

- Three part-time occupational therapists

- Two full-time physiotherapists (one of whom is the team lead)

- Three full-time rehabilitation assistants, (supporting the nurses, occupational therapists, and physiotherapists within the team)

- Six consultant physician sessions (provided by three senior geriatricians)

- Line management through the Trust's clinical manager for intermediate care.

OPAL was a new development that required an annual cost of $£ 407702$ (2010 rates), mainly for staff salaries. All of this was recovered within two years when shorter lengths of stay enabled an off-site decanting ward to be closed, with an overall yearly saving of $£ 850,000$. The contribution of OPAL to the length of stay reduction was estimated at $50 \%$, so the project was approximately costneutral to deliver the intended operational and therapeutic protocol.

Before the introduction of OPAL, elderly patients were cared for by the medical and nursing staff generically responsible for acute admissions. They were then referred on to specialist geriatricians about $24-48$ hours later. There were no physiotherapists or occupational therapists embedded in the admitting areas at that time. 


\section{OPAL modus operandi}

A core operational policy for the OPAL team was, of course, determined during the commissioning stage, although this needed time to reach full function during recruitment and induction, and had been modified to some extent in the light of experience. However, it was fully established and in stable form for the 18-month period leading up to the study. It can be summarized as being to facilitate the safe early discharge of newly admitted older patients with multidisciplinary needs who might otherwise have an unnecessarily long hospital stay.

Referral inclusion criteria are:

- Age 65 years or more

- Currently admitted to the Clinical Decisions Unit, Acute Admissions Ward, Accident and Emergency Department, or Fast Track Rehabilitation Ward

- Medically fit enough to be managed at home or in a community rehabilitation bed

- Likely to have had a change in their functional level as a result of the episode, for example, mobility, personal activities of daily living, etc

- Those who have fallen, particularly recurrent fallers

- Frequent attenders

- Those reporting difficulty managing at home. Referral exclusion criteria were:

- Glasgow Coma Scale $<13$

- Active diarrhea and/or vomiting

- Currently withdrawing from alcohol

- New requirement for oxygen therapy

- New requirement for nebulized bronchodilators

- New requirement for hoist transfers

- Lack of inclusion criteria.

The majority of patients eligible for OPAL were identified during the immediate postadmission senior medical ward rounds. Senior nurses in the acute admissions areas also flagged patients for OPAL review. In addition to the application of the above criteria, it was made clear that in uncertain cases the default position was to refer to OPAL. Further, the OPAL staff trawled the admissions and Accident and Emergency Department attenders daily for potential patients who might have been overlooked for OPAL referral or might have become eligible after initial treatment.

Patients referred to OPAL received a comprehensive geriatric assessment, conducted flexibly with as much crossprofessional working as possible. The emphasis was on extended scope of practice, thoroughness, speed, and focused problem solving. The team was fully staffed Monday-Friday, with reduced staffing at weekends.

\section{Methods}

\section{9 survey of acute admissions}

The first step in the study was to take a sufficiently large sample of acute (all age) medical admissions in 2009 to compare with data from 2003. The sample was taken at the same time of year. No changes had occurred in the formally agreed admission criteria for acute medical admissions between the two samples, and the admission pathway was operationally identical with very few inhouse geographic differences.

The sample target was a minimum of 1000 patients. We took data from all individual medical emergency patients admitted consecutively over 25 days. Those included were all patients listed for post-take consultant ward rounds. This was identical to the selection method used in 2003, and spanned the same number of weekends. Therefore, it included all medical patients admitted to the Clinical Decisions Unit and downstream acute admission facilities, including the Acute Admissions Unit, other wards, Coronary Care Unit, High Dependency Unit, Intensive Care Unit, and other acute settings. Patients reviewed and discharged by on call medical registrars were also included, as were interhospital transfers. Medical patients seen in the Accident and Emergency Department but not referred to the on call medical team were not included in the main survey. The 25-day sample provided far more fine-grained information than could have been obtained from data routinely collected for Trust managerial purposes. Overall annual acute medical admission rates for the Trust were obtained for the 2003-2009 period to place the detailed samples in context.

Data were gathered every day in paper format using a template (Appendix 1) that included the same core items as in 2003, plus information about OPAL involvement. A medically qualified research assistant performed the initial step using the case records, and the extracted information was then reviewed by a senior geriatrician. There was daily discussion to resolve borderline or uncertain classifications. Most of the data were categoric and relatively easy to record. Some follow-up information, such as the date and destination of discharge, was collected later and added to the template.

The descriptors of age, frailty, and comorbidity used were the same as in the 2003 survey, and based on the British Geriatrics Society definitions: ${ }^{3}$

- All people aged 80 years or more

- Patients aged 65-79 years with:

- acute confusion

- falls

- incontinence of urine and/or feces

- dementia

- immobility 
- complex multipathology chronic illness

- social breakdown

- any combination of the above.

\section{Looking closely at effects of OPAL}

Headline data on OPAL activity were obtained from the managerial data set for the corresponding half-year in which the study period fell. The actual number of patients seen daily by the OPAL team during the study period was obtained from the team record. This was available for comparison with other contemporaneous data, including referral rates to the team and case capture by the team from trawling the acute admissions.

We also set out to look in detail at the OPAL pathway for samples of individual patients. We took a random sample of 20 medically admitted patients referred to OPAL during the study period for comparison with a case-matched sample from the 2003 survey. The match criteria were same gender, age within three years, same main diagnosis, same type of preadmission residence, and same preadmission functional status. This was a labor-intensive process that was undertaken by a medical undergraduate with senior supervision. Once the matched samples had been completed, the case records were scrutinized to determine key factors in the individual care pathways. These were:

- Length of stay in hospital

- Time to first review by a senior geriatrician

- Time to first review by a physiotherapist and occupational therapist

- Time to recorded declaration that the patient was medically ready to leave the hospital

- Time to arrange package of care.

\section{Statistical methods}

Most of the data were presented in comparative form using descriptive statistics. Some categoric data were tested using the Yates' test. Some data with normal or near-normal distribution were tested using parametric statistical tests.

\section{Results}

\section{Total acute medical admission rate}

In the 2009 survey there were 1105 admissions in 25 days, compared with 1056 over 30 days in 2003. This was a rise of $25.5 \%$ from 35.2 per day in 2003 to 44.2 per day in 2009 , equivalent to nine more patients per day. The rise detected by the sample is consistent with Trust data showing a $29.1 \%$ increase in total nonelective medical admissions between 2003 and 2009.

\section{Frail elderly admissions}

The proportion of overall admissions meeting the frail and/or elderly category rose from $39.2 \%$ to $44.4 \%$. The number of patients in the frail and/or elderly category had risen from 13.8 per day in 2003 to 19.6 per day in 2009 . This was a $42 \%$ increase within that category, and amounted to an average increase of almost six patients per day. Therefore, of the nine per day overall increase between 2003 and 2009, six (66\%) were in the frail and/or elderly category. The mean age was 83 years (range $65-107,61 \%$ female) in 2003 and 84 years (range 65-104, 60\% female) in 2009.

\section{Triage}

A judgment was made of triage quality. This was by a senior physician using agreed referral criteria. In 2003, 76.8\% had an ideal triage. This had improved to $91.4 \%$ in 2009 . The main reason was probably that new patients were triaged by consultants on post-take ward round in 2009, whereas in 2003 about a third of patients were triaged by a specialist registrar. Further, the proportion that should have been triaged to a geriatrician but were not had improved from $17.1 \%$ to $7.2 \%$; the main reason again was probably consultant triage, but might also be an effect of having daily input via the Acute Admissions Unit by geriatricians. An initial management plan was in place for all patients after the post-take ward round, and in many cases before that.

\section{OPAL}

\section{Headline data}

The six-month sample of headline OPAL data from managerial records included the 25-day period used for the main survey. This confirmed that the daily rate of uptake of patients by OPAL during the survey, discharge rate, and casemix were typical during the survey. This is summarized in Table 1. The two OPAL data sets had a similar mean patient age (84 and 82 years, respectively) and gender ratio (59\% and $62 \%$ female, respectively).

During the survey period the OPAL team were involved with a mean of 10.2 new patients per day (range 3-16). This was about half the frail elderly admissions. Of these, a mean of four per day were flagged at the post-take ward round, and the remainder were discovered on case-trawling by OPAL staff or on referral by Acute Admissions Unit staff. Scrutiny of the medical information gathered for the survey indicated that this combined approach to case finding by OPAL resulted in a $94 \%$ capture of eligible patients. Sixteen percent of those referred did not meet the OPAL referral criteria, although very few were entirely inappropriate because, in most cases, 
Table I Comparison of the six-month headline OPAL data with the survey period OPAL data. The diagnostic categories are for the main presenting problem and do not include comorbidities or functional status. Rates are monthly unless otherwise stated

\begin{tabular}{llll}
\hline & $\begin{array}{l}\text { Six-month } \\
\text { OPAL data }\end{array}$ & $\begin{array}{l}\text { Survey } \\
\text { OPAL sample }\end{array}$ & P \\
\hline Total number & 1755 & 255 & \\
Mean per day & 9.6 & 10.2 & NS \\
Falls (\%) & 40.6 & 36.4 & NS \\
Fracture (\%) & 16.0 & 12.2 & NS \\
Respiratory (\%) & 10.6 & 14.5 & NS \\
Rheumatology (\%) & 12.4 & 11.0 & NS \\
Neurology (\%) & 7.2 & 10.2 & NS \\
Other (\%) & 13.2 & 15.7 & NS \\
Early supported discharge & 29 & 32 & NS \\
after medical admission & & & NS \\
Early supported discharge & 37 & 34 & \\
from Accident and & & & \\
Emergency Department & & & \\
\hline
\end{tabular}

Abbreviations: OPAL, older persons' assessment and liaison; NS, not significant.

the OPAL staff were able to contribute positively to the diagnostic workup or management plan.

The high mean length of stay figures in Table 2 were due to the influence of a small number of patients who had a very prolonged eventual length of stay despite early involvement with OPAL in the 2009 sample (three patients) and in matched comparators from the 2003 sample (four patients). A more accurate insight was gained by looking more closely at the

Table 2 Comparison of selected process indices in the 2009 OPAL sample and matched cases from 2003. This applies to patients seen by OPAL after medical admission, and excludes those seen in the Accident and Emergency Department for whom therapy review occurred within a mean of less than two hours

\begin{tabular}{llll}
\hline & $\begin{array}{l}\text { 2003 matched } \\
\text { sample }(\mathbf{n}=\mathbf{2 0})\end{array}$ & $\begin{array}{l}\text { 2009 patients } \\
\text { referred to } \\
\text { OPAL }(\mathbf{n}=\mathbf{2 0})\end{array}$ & $\mathbf{P}$ \\
\hline $\begin{array}{l}\text { Mean (range) } \\
\text { LOS (days) }\end{array}$ & $14.0(\mathrm{I}-56)$ & $9.6(3-64)$ & $<0.05$ \\
$\begin{array}{l}\text { Mean (range) } \\
\text { time to therapy }\end{array}$ & $2.9(\mathrm{I}-10)$ & $1.7(\mathrm{I}-3)^{*}$ & $<0.05$ \\
$\begin{array}{l}\text { (OT and/or PT } \\
\text { review (days) }\end{array}$ & & & \\
$\begin{array}{l}\text { Mean (range) } \\
\text { time to senior } \\
\text { geriatrician }\end{array}$ & $3.4(\mathrm{I}-7)$ & $2.0(\mathrm{I}-4)$ & $<0.05$ \\
review (days) & & & $\mathrm{NS}$ \\
$\begin{array}{l}\text { Mean (range) } \\
\text { time to first } \\
\text { recorded } \\
\text { readiness for } \\
\text { discharge }\end{array}$ & $7.1(\mathrm{I}-42)$ & $5.3(\mathrm{I}-39)$ & \\
\hline
\end{tabular}

Abbreviations: LOS, length of stay; OT, occupational therapist; PT, physiotherapist; OPAL, older persons' assessment and liaison; NS, not significant.
OPAL patients who had "early supported discharge" after medical admission, most of whom had a length of stay in the 1-3 day range. Furthermore, patients discharged from the Accident and Emergency Department with an early supported discharge after OPAL involvement were not included in the survey of medical admission, and had a mean length of stay of less than four hours.

Thirty-two percent of those seen by OPAL after medical admission were discharged with an early supported discharge arrangement, such as through the Community Assessment and Rehabilitation Team and other community agencies. The community services available had not changed substantially between 2003 and 2009. The remainder had a range of other timely decisions and interventions made, and were directed to the next best destination (such as an acute or fast track rehabilitation ward).

From the comparison of medically admitted OPAL early supported discharge patients during the study period and pre-OPAL matched patients (using the same method as that described above), we found that each early supported discharge patient went home a mean of three days earlier. Therefore, based on the mean number of patients receiving the intervention, we estimated that, at any given time, nine beds were freed by OPAL early supported discharge activity. Furthermore, we found narrative evidence that other OPAL actions, including early consultant review and fast track investigations, probably saved at least one day per patient. Therefore, as a conservative estimate, OPAL activity resulted in a mean daily bed saving of around 12, with a range of nine (assuming maximum effect of other factors) and 16 (assuming minimum effect of other factors). This represented $8 \%-14 \%$ of the bed complement in the combined acute admissions areas.

There was case note evidence of improved diagnostic workup and treatment plans as a result of the input from the geriatricians in the OPAL team. It was not possible to find reliable information to support the comparison of other outcomes. In particular, it was not possible to obtain data to compare the numbers of, and outcomes for, older patients sent home from the Accident and Emergency Department in the pre-OPAL era with those after full implementation of the service. A three-month audit sample showed that the 30-day readmission rate was $7 \%$ for those who received an OPALinitiated early supported discharge. Analysis showed that none of these was due to a failure of service provision, and all were the result of a new illness or unpredictable relapse of existing pathology. No suitable pre-OPAL readmission data were available for comparison. Differentiated mortality data 
to compare in-hospital and out-of-hospital rates for OPAL and pre-OPAL periods were not available.

\section{Avoidance of admission from Accident and Emergency Department}

The mean daily number discharged directly from the Accident and Emergency Department after OPAL involvement was 3.6. It was not possible, for obvious reasons, to be precise about the proportion of these interventions that avoided a medical admission. However, the collective opinion of medical and nursing staff in OPAL and the Accident and Emergency Department indicated that the proportion was about $50 \%$, thus indicating that OPAL in the Accident and Emergency Department prevented approximately one or two medical admissions per day on average.

\section{Discussion}

A number of useful messages emerged from the study. We again demonstrated the power of taking a large detailed sample of medical admissions to provide an information set with sufficient resolution to describe day-to-day operational activity. The questions we posed could not have been answered by routinely recorded data. There were difficulties inherent in attempting to measure the effects of a new service embedded in a complex and changing health care environment. Nevertheless, the combined approach of taking detailed information from case records, activity data recorded by the new service, headline data from Trust information systems, and verbal narrative from the staff enabled us to gain a reliable description of most OPAL processes and some outcomes. Of course, this approach has limitations and cannot claim the credibility of a randomized, parallel, controlled study or even a prospective before-andafter study. Such approaches are, however, rarely possible in a full-scale service context because once needs are identified and resources secured there is normally a managerial expectation of immediate implementation. In such circumstances, our approach of using historic numeric comparators, case-matched controls, and narrative evidence provided information of sufficient robustness to be the basis for forward planning. The need for alternative approaches to assess and describe complex health interventions is now increasingly recognised. ${ }^{4}$
We provided evidence that OPAL is delivering the improvements that were envisaged during commissioning. The timeliness of discharge demonstrated by close analysis of the survey sample was consistent with headline Trust data, and remained positive when tested across a range of assumptions. The detailed scrutiny of individual case journeys showed a significantly earlier involvement of key staff, which can be regarded as an indication of enhanced quality of care as well as a step towards reducing length of stay. Staff perceptions of the benefits of OPAL were uniformly positive from within and outside the OPAL team, and managerial support remained enthusiastic.

Our close look at OPAL has identified key factors that have enabled us to begin transferring the OPAL approach to intervention and discharge planning to other wards and departments. Those factors include early comprehensive geriatric assessment, diagnostic accuracy and completeness, multidisciplinary management for frail elderly patients during admission and for ongoing health care intervention in the community, use of a flexible workforce through extended scope of practice and blurring of professional boundaries, specialist multidisciplinary training in care of the elderly, professional confidence to take calculated risks, determination to overcome treatment and discharge planning barriers, "we can get this done today", a polite but firm approach to external agencies, and individuals who are in no hurry. Over time we intend to embed this mindset across all parts of the Trust where frail older people are treated.

\section{Disclosure}

The authors report no conflicts of interest in this work.

\section{References}

1. Allen SC, Stockdale R, Black T, Raza T. Gathering facts to drive change: Services for acutely ill elderly people presenting to a general hospital. Clinician Manag. 2005;13:53-58.

2. Harari D, Martin FC, Buttery A, Neill S, Hopper A. The older persons assessment and liaison team 'OPAL': Evaluation of comprehensive geriatric assessment in acute medical inpatients. Age Ageing. 2007;36: 670-675.

3. Fry A, Chandle L, Khanna P, Wilkinson M. Developing a frailty pathway for Gwent. BGS Newsletter. 2009;22:15.

4. Campbell M, Fitzpatrick R, Haines A, et al. Framework for design and evaluation of complex interventions to improve health. $B M J$. 2000;321:694-696.
Pragmatic and Observational Research

\section{Publish your work in this journal}

Pragmatic and Observational Research is an international, peer-reviewed, open access journal that publishes data from studies designed to reflect more closely medical interventions in real-world clinical practice compared with classical randomized controlled trials (RCTs). The manuscript

\section{Dovepress}

management system is completely online and includes a very quick and fair peer-review system. 